\title{
Numerical Study of Reactive Flow Past a Wedge in a Channel
}

\author{
Hui-Yuan Fan ${ }^{1}$ and Frank K. Lu ${ }^{2}$ \\ Aerodynamics Research Center, Mechanical and Aerospace Engineering Department, \\ The University of Texas at Arlington, Arlington, TX 76019-0018, USA
}

\begin{abstract}
Detonation of the flow of a combustible mixture over a wedged channel is numerically simulated. A two-dimensional, time accurate, finite-volume-based method is used to perform the computations, and a five-species, two-step chemical reaction is assumed for a stoichiometric hydrogen-air mixture. The combustion channel is made of a wedged section followed by a constant area section. The simulation was performed with wedges of up to 20 deg semi-angle and Mach numbers from 2 to 6.5, with other inflow parameters fixed. Different types of flows arise depending on the wedge angle and the incoming Mach number. Propagating and standing detonation waves modes were found, both of which can be further subdivided, depending on where the detonation is initiated. Moreover, a case without combustion was discovered for a narrow range of Mach numbers for the 5 deg wedge.
\end{abstract}

\section{Introduction}

$\mathrm{W}$ EDGE-induced detonation is an intrinsically unsteady combustion process that encompasses various aerodynamic and chemical phenomena. Such a class of problems has received increasing attention recently because of its potential applications in future hypersonic propulsion devices. For example, a wedge-induced detonation wave may be used in a ram accelerator to accelerate projectiles to very high speeds. ${ }^{1-4}$ Another relevant concept that makes use of detonation waves for propulsion purposes is the oblique detonation wave engine. ${ }^{5-7}$ The idea is to use the thrust from the wedge-induced detonation of a fuel-air mixture. Munipalli et al. ${ }^{8}$ and Wilson et al. ${ }^{9}$ have proposed that unsteady detonation waves can be incorporated into a multimode propulsion system for high-speed flight. Wedge-induced detonation is also a phenomenon of fundamental interest with a rich variety of time and length scales (see, for example, Refs. 10-14). Despite numerous fundamental studies, there are still a lot of poorly understood features.

A serious handicap in understanding confined, wedge-induced detonations is experimental difficulties. However, numerical simulations of wedge-induced detonation waves have been performed by various authors recently. Figueria da Silva and Deshaies ${ }^{15}$ revealed two different kinds of overall flow configurations arising from wedgeinduced detonation waves, namely, direct initiation of a detonation wave within the stagnation region, or an oblique shock wave/oblique detonation wave transition occurring at some distance downstream from the wedge's leading edge.

Most the available studies of wedge-induced detonation waves involve long wedge, that is, the wedges are too long for their top corner to affect the structure of the reaction zone (Papalexandris ${ }^{16}$ ). Also, most studies idealize the flowfield to only a single wedge in a semi-infinite domain, without considering a confined wedge. Such studies explore only a limited range of phenomena. Confining the detonation within a channel unveils a rich variety of phenomena that do not appear to be well explored. The present numerical study intends to explore such phenomena, revealing complex wave reflections that can seriously influence the initiation, transition and propagation waves.

\section{Method}

Numerical simulation of confined, wedge-induced detonation waves were performed to explore the phenomena found in such flows, with particular attention to the wave processes. The numerical method taken to perform the required computations is a two-dimensional, time accurate, finite-volume-based method developed by Kim at al. ${ }^{17}$ A schematic of the configuration is shown in Fig. 1. A two-dimensional channel is formed from a symmetric wedge

\footnotetext{
${ }^{1}$ Research Scientist

${ }^{2}$ Professor, Associate Fellow AIAA
} 
with a straight end-section symmetrically arranged within a straight chamber. The wedge half-angle ranged from 5 to 20 deg. An incoming stoichiometric oxygen, hydrogen and nitrogen mixture at $700 \mathrm{~K}$ and $0.101325 \mathrm{MPa}$ flows past the wedge with Mach numbers ranging from 2 to 6.5 .

\section{A. Model Equations}

The numerical technique was previously reported in Kim et al. ${ }^{17}$ and only a brief overview is provided here. The timedependent two-dimensional Euler equations are used to describe

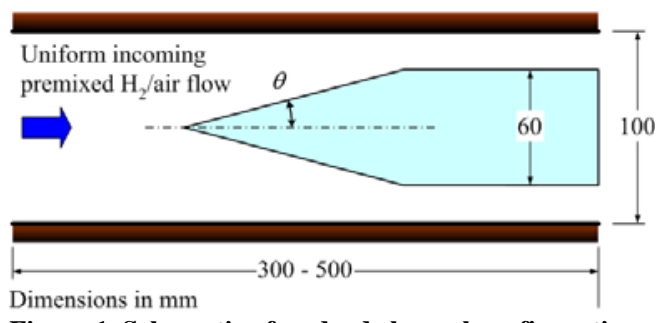

Figure 1. Schematic of wedged channel configuration. an inviscid, non-heat-conducting, reacting gas flow in which thermal non-equilibrium is modeled with a twotemperature model. These equations can be expressed in the Cartesian coordinate as

$$
\frac{\partial U}{\partial t}+\frac{\partial F}{\partial x}+\frac{\partial G}{\partial y}=S
$$

where $U$ is the vector of conserved variables, $F$ and $G$ are the convective flux vectors, and $S$ is the vector of source terms:

$$
U=\left[\begin{array}{l}
\rho_{s} \\
\rho u \\
\rho v \\
\rho e_{v} \\
\rho E
\end{array}\right], F=\left[\begin{array}{l}
\rho_{s} u \\
\rho u^{2}+p \\
\rho u v \\
\rho u e_{v} \\
\rho u E+p u
\end{array}\right], G=\left[\begin{array}{l}
\rho_{s} v \\
\rho u v \\
\rho v^{2}+p \\
\rho v e_{v} \\
\rho v E+p v
\end{array}\right], \quad S=\left[\begin{array}{l}
w_{s} \\
0 \\
0 \\
w_{v} \\
0
\end{array}\right]
$$

The subscript $s=1,2,3, \ldots, N_{s}$ where $N_{s}$ is the number of species. The first $N_{s}$ rows represent species continuity, followed by the two momentum conservation equations for the mixture. The next row describes the rate of change in the vibrational energy and the final row is the total energy conservation equation. The terms $u$ and $v$ are the velocities in the $x$ and $y$ directions respectively, $\rho=\sum_{s=1}^{N_{s}} \rho_{s}$ is the mixture density, $\rho_{s}$ is the density of species $s, p$ is the pressure, $e_{v}$ is the vibrational energy, $E$ is the total energy per unit mass of mixture, $w_{s}$ is the mass of production rate of species $S$ per unit volume, and $w_{v}$ is the vibrational energy source.

The internal energy based on the two-temperature model is assumed to comprise of an equilibium portion at the translational temperature $T$ and a nonequilibium portion at the vibrational temperature $T_{v}$, and can be defined as

$$
e=e_{e q}(T)+e_{v}\left(T_{v}\right)
$$

These energy components can be determined with certain thermodynamic relations.

The source terms for the species mass production rate in the chemical reactions can be written as

$$
w_{s}=M_{s} \sum_{r=1}^{N_{r}}\left(\beta_{s, r}-\alpha_{s, r}\right)\left(R_{f, r}-R_{b, r}\right)
$$

where $M_{s}$ is the molecular weight of species $s, N_{r}$ is the number of reactions, $\alpha_{s, r}$ and $\beta_{s, r}$ are the stoichiometric coefficients for reactants and products, respectively, in the $r$ th reaction. The forward and backward reaction rates of the $r$ th reaction are $R_{f, r}$ and $R_{b, r}$ respectively. These rates can be determined by the Arrhenius law.

The source term of vibrational energy can be written as

$$
w_{v}=\sum_{s} Q_{v, s}+\sum_{s} w_{s} e_{v, s}
$$

The first term on the right hand side, $Q_{v, s}$, represents the vibrational energy exchange rate of species $S$ due to the relaxation process with translational energy, which can be determined by the Landau-Teller formulation. ${ }^{18,19}$ The second term, $w_{s} e_{v, s}$, represents the amount of vibrational energy gained or lost due to production or depletion of species $S$ from chemical reactions.

A finite-volume algorithm was used to solve these equations numerically. The advantage of this method is its use of the integral form of the equations, which ensures conservation and which allows for the correct treatment of 
discontinuities. Nonequilibrium flows involving finite-rate chemistry and thermal energy relaxation often can be very difficult to solve numerically because of stiffness. The present method includes a point implicit treatment of source terms to reduce the inherent stiffness of the system by effectively rescaling all the characteristic times in the fields into the same order of magnitude. Roe's flux-difference split scheme is combined with the Runge-Kutta integration schemes for second-order accuracy in capturing the shock waves in space and time.

In the current study, the Rogers-Chinitz ${ }^{20}$ hydrogen-air combustion mechanism of 5-species $\left(\mathrm{N}_{2}, \mathrm{O}_{2}, \mathrm{H}_{2}, \mathrm{H}_{2} \mathrm{O}\right.$ and $\mathrm{OH}$ ) and 2-reactions $\left(\mathrm{H}_{2}+\mathrm{O}_{2}=2 \mathrm{OH}\right.$ and $\left.2 \mathrm{OH}+\mathrm{H}_{2}=2 \mathrm{H}_{2} \mathrm{O}\right)$ is used. This model was developed to represent hydrogen-air chemical kinetics with as few reaction steps as possible while still giving reasonably accurate global results. In this model, nitrogen is counted as a collisional partner in the thermodynamic model and relaxation process, but not included in the chemical reaction model since the maximum temperature in the hydrogen-air reaction does not reach the dissociation temperature of nitrogen.

\section{B. Configuration and Flow Conditions}

The upper half wedged channel is taken as the computation domain. The configuration, as shown in Fig. 1, comprises of a sharp wedge with half angle $\theta$ from 5 through 20 deg. The sharp wedge, pointing upstream, is $60 \mathrm{~mm}$ wide and is attached to an aftbody, $100 \mathrm{~mm}$ long, and of the same width as the base of the wedge. The entire body is placed in a channel $100 \mathrm{~mm}$ wide. The length of the computational domain varies from 300 through $500 \mathrm{~mm}$ depending on $\theta$. This variation is to ensure that the clearance between the wedge tip and channel inlet remains approximately constant. The incoming supersonic flow comprises a premixed stoichiometric hydrogen-air mixture. The pressure and temperature of the flow are fixed at $p_{\infty}=0.101325 \mathrm{MPa}$ and $T_{\infty}=700 \mathrm{~K}$ respectively while the incoming Mach number is allowed to vary from 2 through 6.5. The matrix of test cases is shown in Fig. 2. The figure also shows various flow domains which will be discussed later.

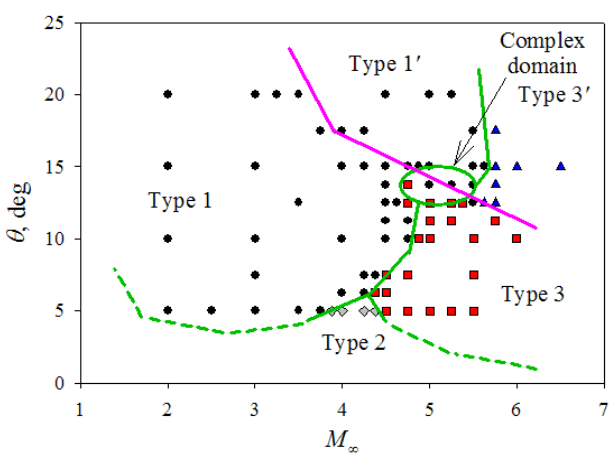

Figure 2. Matrix of test cases and flow domains; $1-$ propagating detonation wave/shock wave mode, 1'propagating detonation wave/shock wave mode with wedge tip initiation, 2-no combustion mode, 3standing detonation wave/shock wave mode, 3'standing detonation wave/shock wave mode with wedge tip initiation.

The different parts of the computational domain are meshed with structured grids, which are not all identical. For example, $25 \times 150$ cells are used for the 5-deg half-angle wedge, whereas $25 \times 100$ mesh cells are used for 15deg half-angle wedge. The flow solver time step in simulation is $10^{-7} \mathrm{~s}$ which is deemed capable of resolving the timescales of interest.

\section{Results and Discussion}

Detonation initiation is accompanied by a deflagration-to-detonation transition (DDT) unless the initiation energy is sufficiently high. ${ }^{21}$ The present simulations show that the subsequent flow transition rapidly to the Chapman-Jouguet (CJ) state ( $p_{C J}=0.665 \mathrm{MPa} ; T_{C J}=3014 \mathrm{~K}$; and $\left.D_{C J}=1927 \mathrm{~m} / \mathrm{s}\right)$. Our previous work using the same numerical technique suggests that a transition phenomenon can be captured. ${ }^{17,22}$ The rapid transition in the present simulations supports the experimental observation by Lu and Wilson ${ }^{23}$ that shock-induced detonation occurs almost instantly and seems to indicate that a direct initiation is possible with shock-induced detonation. In the present study, we propose the possibility that the impingement of an oblique shock initiates combustion. If the oblique shock is weak, combustion occurs after one or a number of shock reflections. The simulations indicate that the combustion rapidly transits to detonation. For brevity, most of the discussion is for $\theta=5$ and $15 \mathrm{deg}$.

\section{A. Five Deg Half-Angle Wedge}

Three different modes were observed for flow past a wedge with a 5 deg half-angle, depending on the incoming Mach number. When $M_{\infty} \leq 3.875$, a Type 1, "propagating detonation wave" mode is obtained in which the initiated detonation wave always propagates upstream. Detonation is initiated at the impingement of an oblique shock after multiple shock reflections. A number of shock reflections are needed to compress the reactive mixture to cause it to detonate. Unlike observations of propagating detonations in a constant-section tube where an entire detonation front forms, the present results shows that detonation initiates at the impingement of an oblique shock. For example, Fig. 3 shows the beginning of a detonation kernel on the aftbody at $t=0.4275 \mathrm{~ms}$ after six reflections. The evolution of the kernel is shown in finer detail in Fig. 4(a). This figure shows that the detonation initiates just downstream of the 


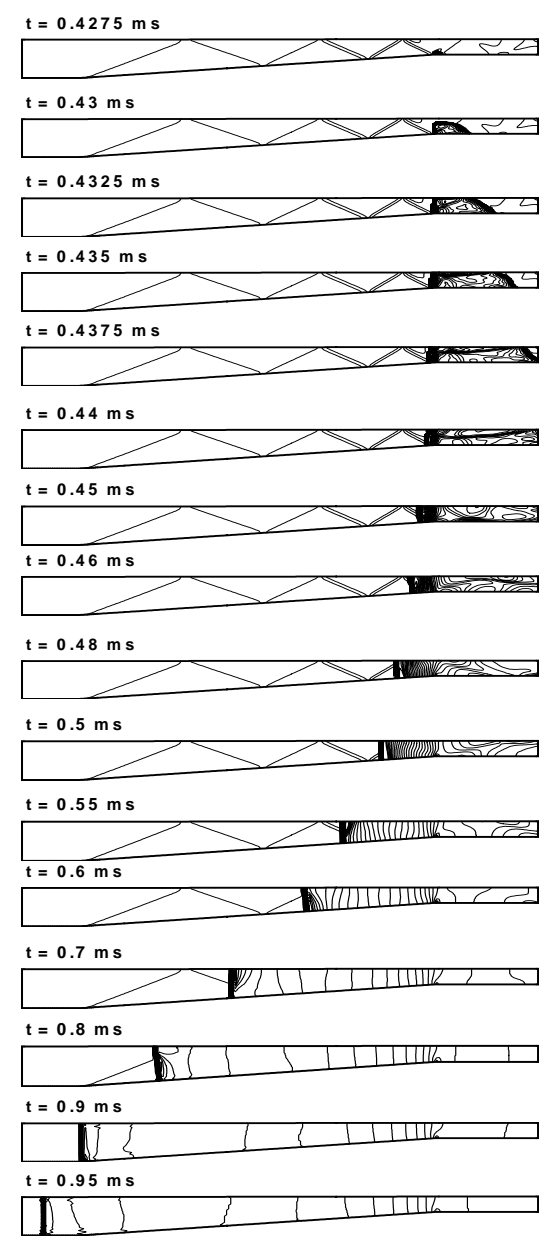

Figure 3. Evolution of detonation wave for $M_{\infty}=3$ and $\theta=5$ deg (Type 1); this and subsequent figures are pressure contours plots.

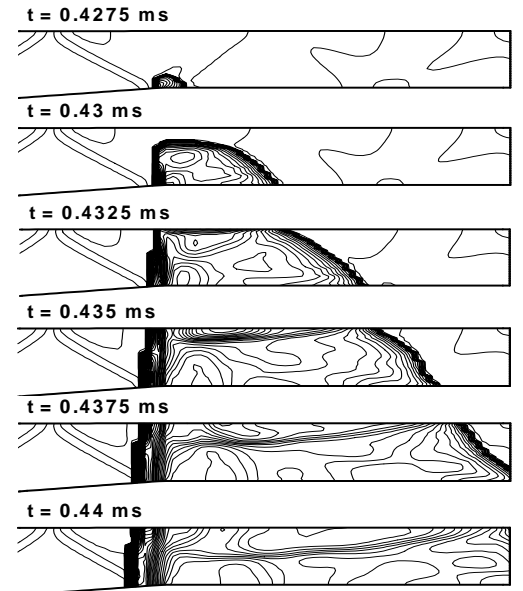

(a) $M_{\infty}=3$

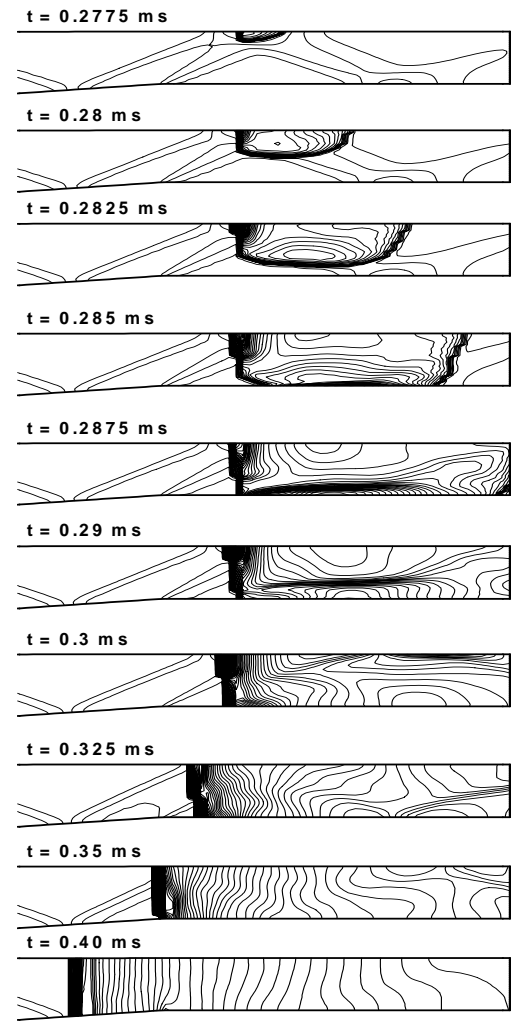

(b) $M_{\infty}=3.5$

Figure 4. Evolution of detonation wave in its initiation phase $M_{\infty}=3$ and 3.5 for $\theta=5 \mathrm{deg}$.

wedge shoulder. However, there are instances where the detonation is initiated on the channel wall. This is shown in Fig. 4(b). In summary, due to the large number of reflections required to trigger the detonation, detonation occurs some distance downstream, either on the surface of the aftbody just downstream of the wedge shoulder or on the channel wall.

Returning to Fig. 3, the detonation kernel grows to fill the entire width of the channel by $0.4325 \mathrm{~ms}$ and is elongated as its downstream propagating front is swept by the incoming flow. However, the upstream front encounters the incoming flow and propagates at a slower rate. As this upstream detonation front propagates past the wedge, it 


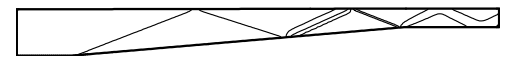

(a) $M_{\infty}=3.875, t \geq 0.38 \mathrm{~ms}$

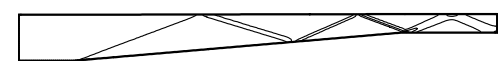

(b) $M_{\infty}=4, t \geq 0.36 \mathrm{~ms}$

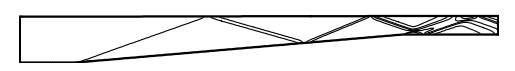

(c) $M_{\infty}=4.25, t \geq 0.33 \mathrm{~ms}$

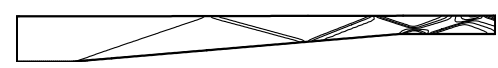

(d) $M_{\infty}=4.375, t \geq 0.31 \mathrm{~ms}$

Figure 5. Final standing shock wave pattern at four incoming Mach numbers for $\theta=5$ deg (Type 2). encounters the steady, oblique shock system. A complex $\lambda$-foot structure forms as can be seen, for example, at $t=0.7$ and $0.8 \mathrm{~ms}$. Eventually, this upstream detonation front propagates out of the left computational boundary normal to the incoming flow.

When $3.875<M_{\infty}<4.5$, the simulation exhibits a steady shock system forms without any combustion occurring in the channel. In other words, the incoming flow, despite multiple shock reflections, cannot ignite the reactants. A detailed mapping shows that this region of no combustion, labeled Type 2, occurs for the narrow Mach numbers range. Moreover, there also appears to be a threshold in the value of $\theta$ below which ignition also does not occur. This entire region is indicated by a dashed boundary in Fig. 2. In Type 2 flows, thermodynamic parameters behind the final oblique shock within the channel, despite multiple reflections, remain lower that the CJ state. The flow generated by the oblique shock system approaches a steady state, as shown for four cases in Fig. 5.

When $M_{\infty}>4.875$, a Type 3, "standing detonation/shock wave" mode occurs. The standing wave structures for five cases in this region are shown in Fig. 6. For each case, the flow in the entire computational domain and an enlargement of the downstream region are shown. For example, at: $M_{\infty}=4.5$, the flow is stabilized at $t=0.45 \mathrm{~ms}$, with the time to achieve stabilization decreasing with increasing Mach number. The enlarged view shows a normal detonation front stretching from the aftbody to the channel wall. Downstream of the normal detonation front are series of shocks and expansions reflecting from both surfaces. In some cases, for example, at $M_{\infty}=5.25$ and 5.5, the standing detonation wave is mostly normal to the incoming flow but exhibits a $\lambda$ structure. Part of the detonation wave interacts with the Prandtl-Meyer expansion at the wedge shoulder and is deflected downstream. This is particularly noticeable in Fig. 6(e).

A more detailed sequence of events for the $M_{\infty}=4.5$ and 5 cases are shown in Fig. 7. Figure 7(a) of the $M_{\infty}=4.5$ case shows ignition on the aftbody surface a short distance after shock reflection, similar to Type 1, the difference being that eventually the Type 3 flow stabilizes into a steady state. Figure 7 (b) for $M_{\infty}=5$ case, on the other hand, shows ignition on the channel wall. This process is more complicated that that of Fig. 7(1) in that the impinging shock at $t=0.1275 \mathrm{~ms}$ is still not stabilized and in moving upstream. This movement appears to induce the ignition and detonation zone to move forward $(t=0.165 \mathrm{~ms})$. Subsequently, a normal detonation front forms and stabilizes at $t=0.35 \mathrm{~ms}$.

\section{B. Fifteen Deg Half-Angle Wedge}

Simulations for $\theta=15$ deg included a range of Mach numbers from 2 through 6.5. In all these cases, a detonation wave is induced on the channel or aftbody surface. This is different from the $\theta=5$ deg cases where detonation occurs only on the aftbody surface if at all. The detonation/shock wave modes can also be divided into propagating and standing types at a Mach number threshold of approximately 5.75 . In addition, the detonation in the $\theta=15$ deg cases can be induced at the wedge tip when $M_{\infty}>$ 4.5. Thus, three modes (Types 1, 1' and 3 ' can be obtained when $\theta=15$ deg as described below.

When $M_{\infty} \leq 4.5$, as typified in Fig. 8, the detonation is initiated downstream of

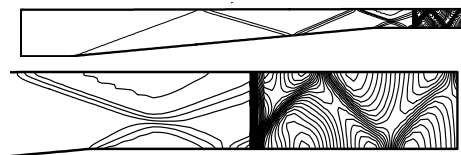

(a) $M_{\infty}=4.5, t \geq 0.45 \mathrm{~ms}$

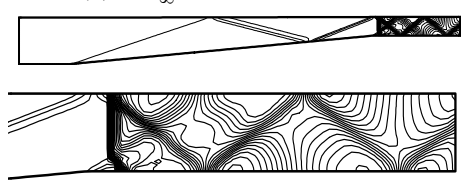

(c) $M_{\infty}=5, t \geq 0.35 \mathrm{~ms}$

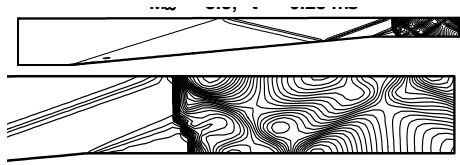

(e) $M_{\infty}=5.5, t \geq 0.25 \mathrm{~ms}$

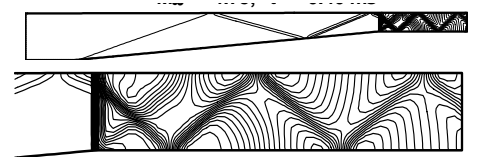

(b) $M_{\infty}=4.75, t \geq 0.4 \mathrm{~ms}$

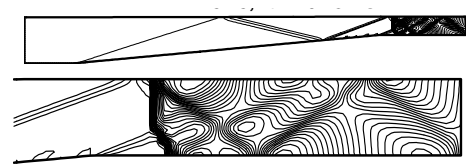

(d) $M_{\infty}=5.25, t \geq 0.28 \mathrm{~ms}$
Figure 6. Final standing detonation and shock wave structures at five incoming Mach numbers for $\theta=5$ deg (Type 3). 


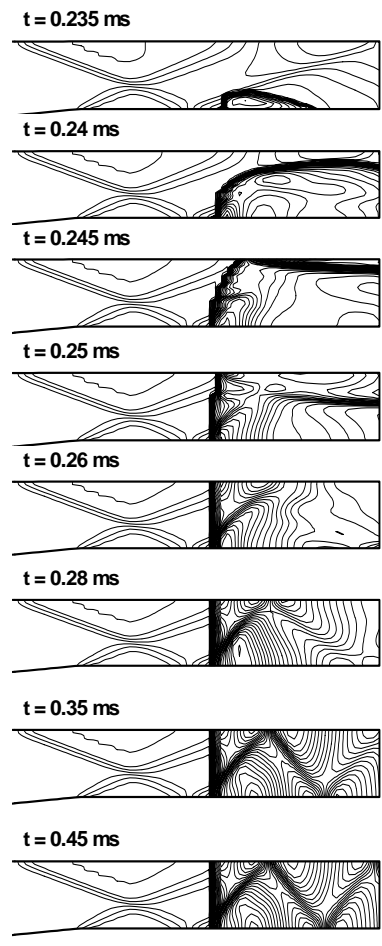

(a) $M_{\infty}=4.5$
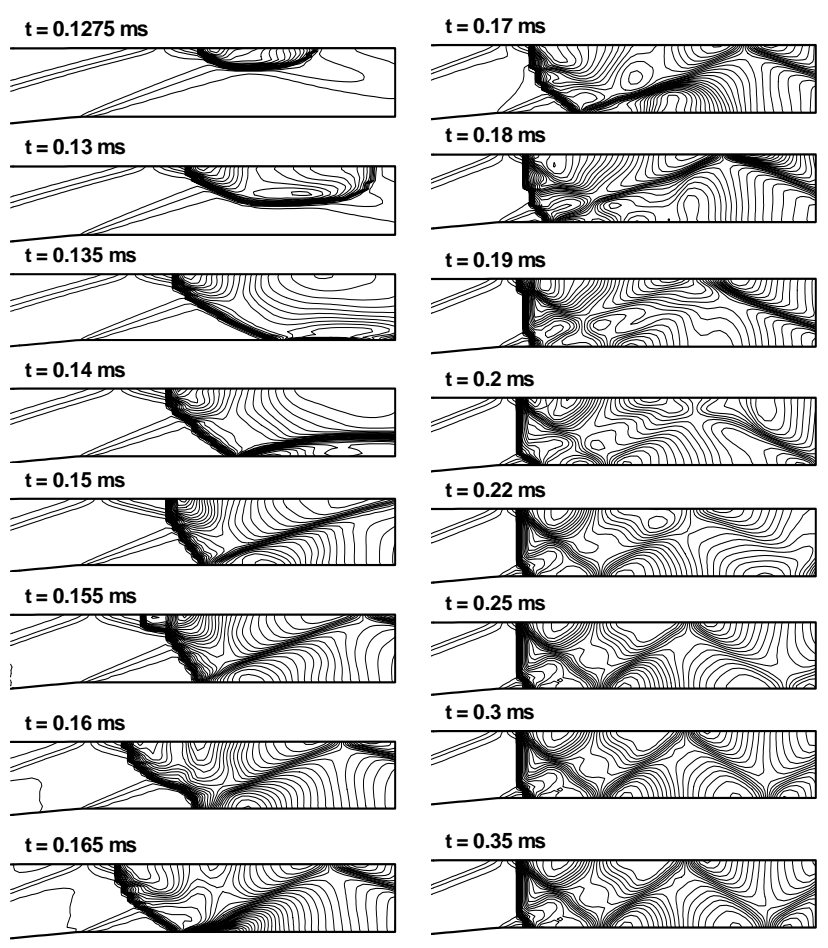

(b) $M_{\infty}=5$

Figure 7. Evolution of detonation wave at $M_{\infty}=4.5$ and 5 for $\theta=5 \mathrm{deg}$

the wedge shoulder and continuously propagates upstream. This mode belongs to the Type 1 mode mentioned previously (Fig. 3). However, the wave evolution in the 15 deg cases is more complex than in the 5 deg cases. The detonation initiates where an oblique shock impinges the aftbody wall, as can be seen at $t=0.065 \mathrm{~ms}$ in Fig. 8 . The detonation wave is further strengthened by subsequent reflection on the opposite wall, followed by its upstream front to form an oblique detonation front at $t=0.075 \mathrm{~ms}$. Meanwhile, the downstream front exits from the channel outlet. The established oblique detonation front evolves into a normal front by $t=0.115 \mathrm{~ms}$. At $t=0.1175 \mathrm{~ms}$, another detonation wave is initiated ahead of this upstream propagating detonation front. It is thought that the "precursor" detonation wave is triggered by the strengthening of the upstream oblique shock from the upstream propagating detonation front. This precursor detonation wave does not completely consume the reactants, thereby allowing the downstream detonation front to exist. A complex shock-detonation wave is formed which continues to propagate upstream through the wedge region to the channel inlet. While Fig. 8 shows a detonation wave triggered on the aftbody at $t=0.065 \mathrm{~ms}$, the present parametric study also revealed that the detonation can be triggered from the channel wall.

When $M_{\infty}=4.5-6.5$, the detonation wave initiates at the tip of the wedge, inducing either a propagating or a standing mode. A propagating mode, labeled Type 1', occurs when $4.5<M_{\infty}<5.5$; otherwise, the wedge induces a Type 3' standing detonation/shock wave structure. In these modes, the detonation initiates at the tip of the wedge that promptly develops over the whole wedge surface. The detonation front expands continuously, reflected by the opposite channel wall, to form an oblique detonation front that attaches at the wedge tip. Behind the oblique detonation wave, shock reflections and expansion waves can developed to produce either Type 1' or 3' flow domains. Figure 9 shows the evolution of the former mode at $M_{\infty}=5$. In this mode, the downstream reflection shock waves induced by the detonation wave move upstream, resulting in a new normal shock wave that overtakes the existing oblique detonation front and as a new detonation front to propagate upstream. Figure 10 shows the evolution the Type 3' flow for $M_{\infty}=6$. The figure shows that the downstream shock reflections induced by the detonation wave is eventually stabilized.

It is worth noting that the Mach reflection phenomenon can be observed for the propagating Types 1 and 1' mode. For example, in Figs. 8 and 9, when the detonation front moves over the oblique shock attached at the wedge 


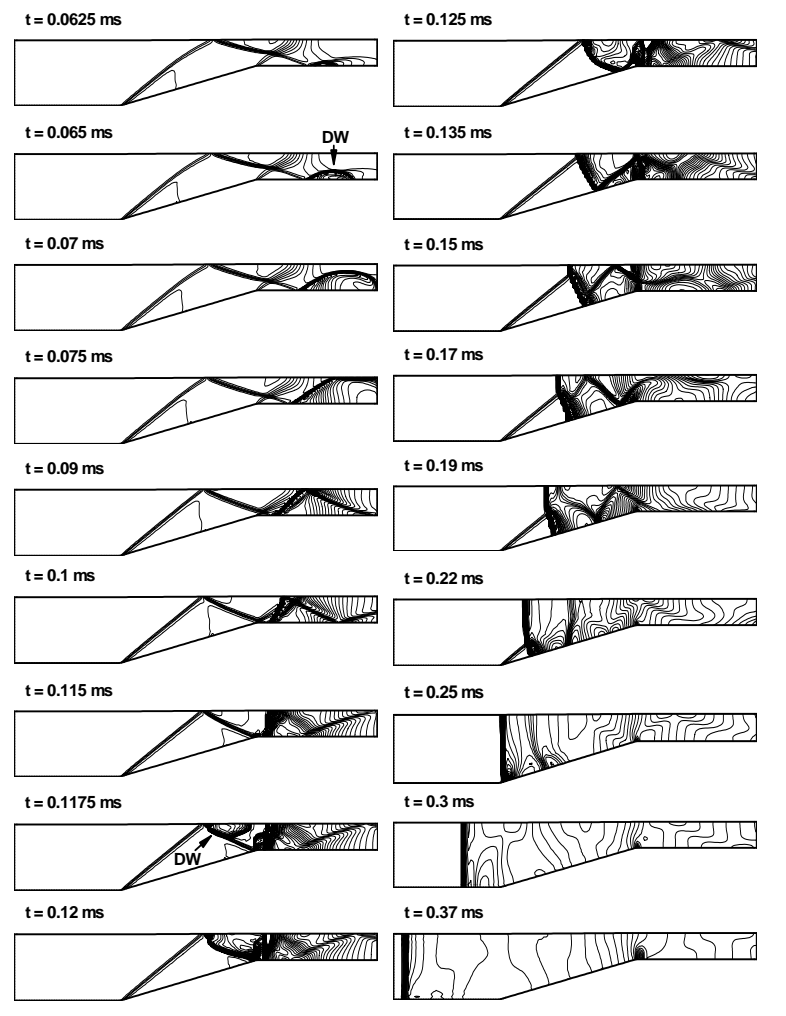

Figure 8. Evolution of detonation wave at $M_{\infty}=3$ for $\theta=15$ deg (Type 1)

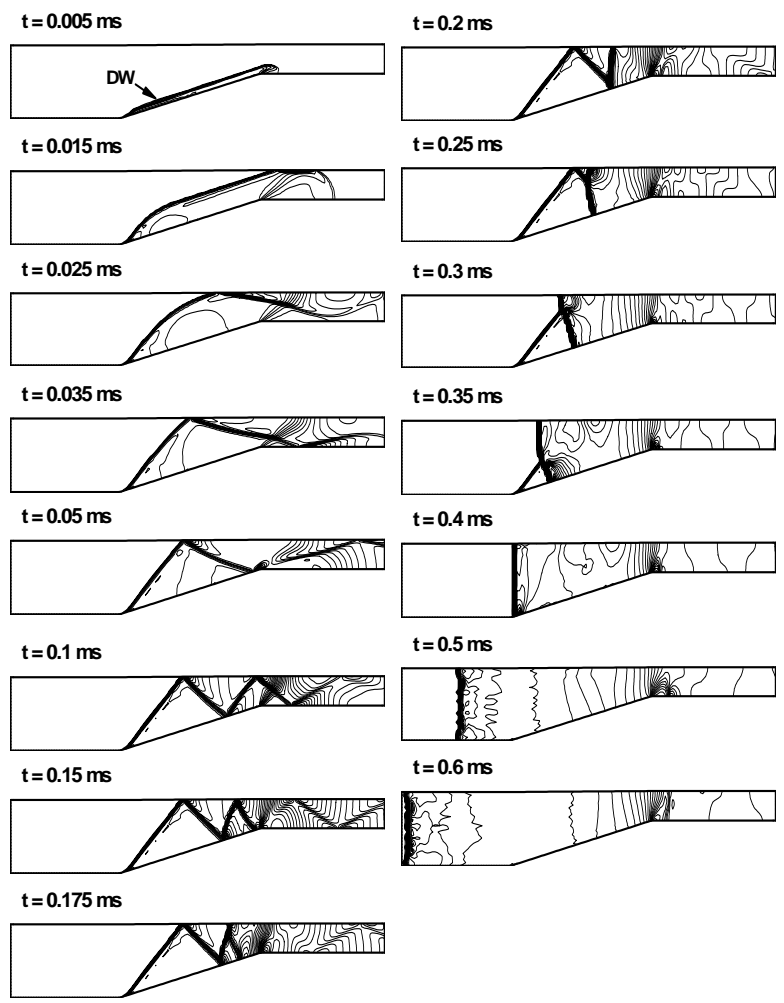

Figure 9. Evolution of detonation wave at $M_{\infty}=5$ for $\theta=15 \mathrm{deg}$ (Type 1')

tip, a Mach reflection configuration appears. In addition, the Type 1' mode in Fig.9 shows the intrinsic instability of a normal propagating detonation front that is usually encountered in a detonation front propagating in channel (see $t=0.6 \mathrm{~ms})$.

\section{Larger Wedge Angles and “Complex Area" in} Fig. 2

Due to limitations of the current code, the simulation the $\theta=20$ deg cases only can be performed for $M_{\infty}<5.25$ and only Type 1 and 1' modes are obtained for these cases. Further, as is presented in Fig. 2, there is the complex area in which the detonation modes may be propagating or standing. The simulation results show that either a propagating or a standing mode can occur here. We hypothesize that this is a transition region between the two major modes. Further work is needed to explore and define this transition region over a larger range of wedge angles.

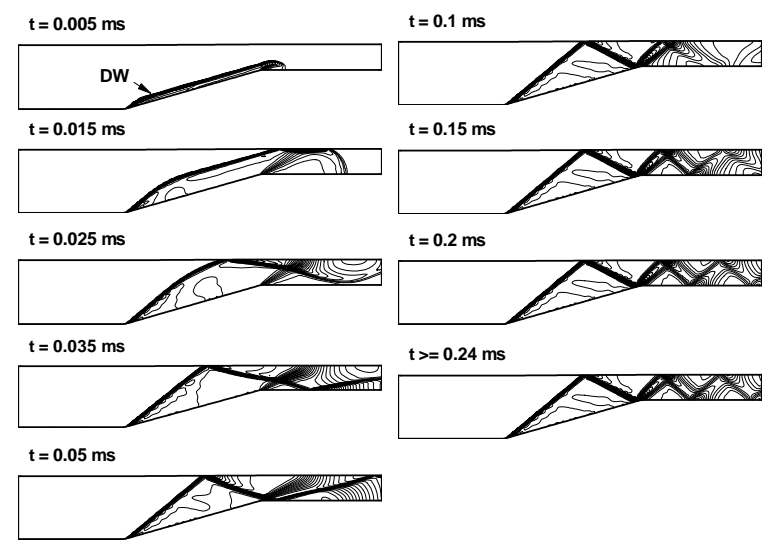

\section{Conclusions}

The wedge-induced detonation occurring in a two-

Figure 10. Evolution of detonation wave at $M_{\infty}=6$ for $\theta=15$ deg (Type 3') dimensional symmetric wedged channel was studied numerically. A five-species and two-step reaction mechanism was adopted to model the thermo-chemical dynamics of the detonation processes and a time-accurate and finitevolume-based method was used to numerical simulate the processes. Half-wedge angles of up to 20 deg were considered. The incoming Mach number was allowed to vary while the other inflow aerodynamic parameters were 
fixed. A qualitative understanding was obtained of the complex and rich detonation flow. Different flow domains arise depending on the incoming Mach number and the wedge angle. Propagating and standing detonation waves were found, both of which can be further subdivided depending on where the detonation is initiated, such as on the channel wall or along the wedge surface, after multiple reflections or at the wedge tip. It was thought that a transitional region exists between these two modes. Finally, a no-combustion case was discovered for a narrow range of Mach numbers for the 5-deg half-angle wedge. This case occurs between the propagating mode at a lower Mach number and a standing mode at a higher Mach number. It was thought that the geometry is such that the shock reflections could not induce a detonation within the domain.

\section{References}

${ }^{1}$ Grismer, M. J. and Powers, J. M., “Calculations for Steady Propagation of a Generic Ram Accelerator Configuration,” AIAA Journal of Propulsion and Power, Vol. 11, No. 1, 1995, pp. 105-111.

${ }^{2}$ Lefebvre, M. H. and Fujiwara, T., "Numerical Modeling of Combustion Processes Induced by a Supersonic Conical Blunt Body," Combustion and Flame Vol. 100, Nos. 1-2, 1995, pp. 85-93.

${ }^{3}$ Hertzberg, A., Bruckner, A. P. and Bogdanoff, D. W., "Ram Accelerator: A New Chemical Method for Accelerating Projectiles to Ultrahigh Velocities,” AIAA Journal, Vol. 26, No. 2, 1988, pp. 195-203.

${ }^{4}$ Kailasanath, K., "Recent Developments in the Research on Pulse Detonation Engines," AIAA Journal, Vol. 41, No. 2, 2003, pp. 145-159.

${ }^{5}$ Brackett, D. C. and Bogdanoff, D. W., "Computational Investigation of Oblique Detonation Ramjet-in-Tube Concepts,” AIAA Journal of Propulsion and Power, Vol. 5, No. 3, 1989, pp. 276-281.

${ }^{6}$ Powers, J. M. and Stewart, D. S., "Approximate Solution for Oblique Detonation in Hypersonic Limit," AIAA Journal, Vol. 30, No. 3, 1992, pp. 726-736.

${ }^{7}$ Terao, K., Ishii, K., Totsuka, T. and Ishikawa, Y., "An Experimental Investigation of Hypersonic Combustion for Ram Jet Engine Applying Detonation Waves,” AIAA Paper 2002-5164, 2002.

${ }^{8}$ Munipalli, R., Shankar, V., Wilson, D. R., Kim, H., Lu, F. K. and Hagseth, P. E., “A Pulsed Detonation Based Multimode Engine Concept,” AIAA Paper 2001-1786, 2001.

${ }^{9}$ Wilson, D. R., Lu, F. K., Kim, H. and Munipalli, R., "Analysis of a Pulsed Normal Detonation Wave Engine Concept,” AIAA Paper 2001-1784, 2001.

${ }^{10}$ Thaker, A. A. and Chelliah, H. K., "Numerical Prediction of Oblique Detonation Wave Structures Using Detailed and Reduced Reaction Mechanisms," Combustion Theory and Modeling, Vol. 1, No. 4, 1997, pp. 347-376.

${ }^{11}$ Grismer, M. J., and Powers, J. M., "Numerical prediction of oblique detonation stability boundaries," Shock Waves, Vol. 6, No. 3, 1996, pp. 147-156.

${ }^{12}$ Powers, J. M. and Gonthier, K. A., "Reaction Zone Structure for Strong, Weak Overdriven, and Weak Underdriven Oblique Detonations," Physics of Fluids A, Vol. 4, No. 9, 1992, pp. 2082-2089.

${ }^{13}$ Ohyagi, S., Obara, T., Nakata, F. and Hoshi, S., "A Numerical Simulation of Reflection Processes of a Detonation Wave on a Wedge,” Shock Waves, Vol. 10, No. 3, 2000, pp. 185-190.

${ }^{14}$ Guo, C., Zhang, D. and Xie, W., "The Mach Reflection of a Detonation Based on Soot Track Measurements," Combustion and Flame, Vol. 127, No. 3, 2001, pp. 2051-2058.

${ }^{15}$ Figueira da Silva, L. F. and Deshaies, B., "Stabilization of an Oblique Detonation Wave by a Wedge: A Parametric Numerical Study,” Combustion and Flame, Vol. 121, Nos. 1-2, 2000, pp. 152-166.

${ }^{16}$ Papalexandris, M., "A Numerical Study of Wedge-Induced Detonation,” Combustion and Flame, Vol. 120, No. 4, 2000, pp. 526-538.

${ }^{17}$ Kim, H., Lu, F. K., Anderson, D. A. and Wilson, D. R., "Numerical Simulation of Detonation Process in a Tube,” Computational Fluid Dynamics Journal, Vol. 12, No. 2, 2003, pp.227-241.

${ }^{18}$ Millikan, R. C. and White, D. R., "Systematics of Vibrational Relaxation," Journal of Chemical Physics, Vol. 39, No. 12, 1963, pp. 3209-3213.

${ }^{19}$ Vincenti, W. G. and Kruger, C. H., Introduction to Physical Gas Dynamics, Krieger, Malabar, Florida, 1997.

${ }^{20}$ Rogers, R. C. and Chinitz, W., "Using a Global Hydrogen-Air Combustion Model in Turbulent Reacting Flow Calculations,” AIAA Journal, Vol. 21, No. 4, 1983, pp. 586-592.

${ }^{21}$ Knystautas, R.,and Lee, J. H. S., "On the Effective Energy for Direct Initiation of Detonations," Combustion and Flame, Vol. 27, 1976, pp. 221-228.

${ }^{22}$ Fan, H. Y. and Lu, F. K., "Comparison Study of Detonation Processes in a Variable Cross-Section Chamber and a Simple Tube," AIAA Journal of Propulsion and Power, 2004, (to be published).

${ }^{23} \mathrm{Lu}$, F. K. and Wilson, D. R., "Detonation Driver for Enhancing Shock Tube Performance," Shock Waves, Vol. 12, No. 6, 2003, pp. 457-468. 